J. BURBEA

KODAI MATH. J.

5 (1982), 339-354

\title{
NORM INEQUALITIES OF EXPONENTIAL TYPE FOR HOLOMORPHIC FUNCTIONS
}

\author{
By JaCOB BurbeA
}

\begin{abstract}
Let $\Delta_{\rho}=\{z \in \boldsymbol{C}:|z|<\rho\}$ with $\rho=1, \infty$ and let $H\left(\Delta_{\rho}\right)$ stand for the class of holomorphic functions in $\Delta_{\rho}$. Let $\phi \in H\left(\Delta_{\rho}\right)$ with $\Delta_{\rho}$ being the domain of holomorphy of $\phi$ and $\phi(0)=0, \phi^{(n)}(0)>0$ for $n=1,2, \cdots$. Then $k(z, \zeta)$ $\equiv \phi(z \bar{\zeta})$ is the reproducing kernel of a uniquely determined Hilbert space $H_{\phi}$ of functions $f \in H\left(\Delta_{\rho}\right)$ with $f(0)=0$ and norm $\|f\|_{\phi}$. The function $\phi \equiv \exp \phi$ also determines a unique Hilbert space $H_{\psi}$ of functions $g \in H\left(\Delta_{\rho}\right)$ with norm $\|g\|_{\psi}$ and such that $K(z, \zeta) \equiv \phi(z \bar{\zeta}), z, \zeta \in \Delta_{\rho}$, is its reproducing kernel. The following is proved: Let $f \in H_{\phi}$, then $\exp f \in H_{\phi}$ and

$$
\|\exp f\|_{\phi}^{2} \leqq \exp \|f\|_{\phi}^{2}
$$

with equality if and only if $f$ is of the form $f(z)=k(z, \zeta)=\phi(z \bar{\zeta})$ for some $\zeta \in \Delta_{\rho}$. The method of proof of this sharp inequality is based on ideas of both Aronszajn and Milin, and it can be extended by replacing the exponential function by any entire function with non-negative Taylor-coefficients. We also give several applications of this inequality in the theory of entire functions and functions holomorphic in the unit disk.
\end{abstract}

\section{Introduction.}

Let $\Lambda$ be an abstract non-void set and let $k(\cdot, \cdot)$ be a scalar-valued kernel on $\Lambda \times \Lambda$. For simplicity, we always assume that the underlying scalar-field is the complex-field $\boldsymbol{C}$. We also assume that $k(\cdot, \cdot)$ is a positive-definite kernel on $\Lambda \times \Lambda$; that is

$$
\sum_{m, n=1}^{N} k\left(z_{m}, z_{n}\right) \alpha_{m} \bar{\alpha}_{n} \geqq 0
$$

for any finite set $\left\{z_{m}\right\}_{m=1}^{N}$ of points of $\Lambda$ and any corresponding complex numbers $\left\{\alpha_{m}\right\}_{m=1}^{N}$. As is well-known, this condition is equivalent to the existence of a uniquely determined Hilbert space $H_{k}$ of functions on $\Lambda$ and admitting $k(\cdot, \cdot)$ as a reproducing kernel, namely

Part of this research was completed while the author was visiting the Mittag-Leffler Institute, Sweden, during 1981-82.

Recelved May 27, 1981 


$$
f(z)=(f, k(\cdot, z))_{k}
$$

for any $z \in \Lambda$ and every $f \in H_{k}$ (see Aronszajn [1]).

Given any entire function $F$ with non-negative Taylor coefficients, we consider the new kernel $K(\cdot, \cdot)=F[k(\cdot, \cdot)]$. This kernel, in view of Schur's theorem, is clearly positive-definite on $\Lambda \times \Lambda$ and hence, as before, it is the reproducing kernel of a uniquely determined Hilbert space $H_{K}$ of functions on $\Lambda$. The structure of this Hilbert space $H_{K}$ involves tensor-products of $H_{k}$ and is rather complicated (see Aronszajn [1], Burbea [4] and Saitoh [9]). The following, however is known (see Burbea [4]): Assume that $f \in H_{k}$, then $F \circ f \in H_{K}$ and

$$
\|F \circ f\|_{K}^{2} \leqq F\left(\|f\|_{k}^{2}\right)
$$

with equality holding if $f=k(\cdot, \zeta)$ for some $\zeta \in \Lambda$.

The equestion of the necessity of the above condition in the equality statement requires, in general, some additional assumptions on $F$ and on both $\Lambda$ and $k(\cdot, \cdot)$. For example, the proof provided in this paper shows that this condition is necessary if the entire function $F$ has positive Taylor coefficients, $\Lambda$ is $\Delta_{\rho}$ $\equiv\{z \in C:|z|<\rho\}, \rho=1, \infty$ and $k(z, \zeta)=\phi(z \bar{\zeta}), z, \zeta \in \Delta_{\rho}$ where $\phi$ is holomorphic in $\Delta_{\rho}$, with $\Delta_{\rho}$ being its domain of holomorphy, and $\phi(0)=0, \phi^{(n)}(0)>0$ for $n=1,2, \cdots$. In this paper, for simplicity and clarity, we treat only the typical case of $F(t) \equiv \exp (t)$. The method of the present proof is based on ideas of both Aronszajn [1] and Milin [6] (see also Pommerenke [8, pp. 78-88]). This results in a rather general theorem which is applicable in the theory of entire functions and functions holomorphic in the unit disk. This sharp inequality will also enable us to provide a shorter proof for a similar inequality on holomorphic functions in the unit disk. The latter was previously proved by us in [4]. A more special case of it was first proved by Saitoh [9], but his proof is rather difficult and involved.

In this paper we also introduce and study, in light of the above inequality, several concrete reproducing spaces, as the so called "generalized Fischer-spaces" and "generalized Hardy-spaces". This study forms an extension of the examples previously developed by us [3] in connection with the total positivity of reproducing kernels. The ordinary Fischer and Hardy spaces were also studied by several authors (cf. [2], [7] and [10]) from various points of view. These spaces have an intimate connection with quantum theory and as such have attracted some attention from physicists (see, for example, Bargmann [2]). They also have connection with the coefficient estimates for univalent functions $[6,8,9]$. The results here can be also extended to cover the case of several complex variables but we shall not pursue this here (see, however, Burbea [5]).

\section{$\S 2$. Spaces of Square Summable Series.}

In this paper $H(D)$ stands for the class of all holomorphic functions in a domain $D$ of the complex plane $C$ while $\Delta$ designates the unit disk in $\boldsymbol{C}$. We 
shall also use the notation $\Delta_{\rho}=\{z \in C:|z|<\rho\}$ with $\rho=1, \infty$ and thus $\Delta_{1}=\Delta$ while $\Delta_{\infty}=\boldsymbol{C}$. Let

$$
\phi(z)=\sum_{n=1}^{\infty} c_{n} z^{n} \quad c_{n}>0, \quad n \geqq 1,
$$

be holomorphic in $\Delta_{\rho}$ and having $\Delta_{\rho}$ as its disk of convergence. We write

$$
H_{\phi}=\left\{f \in H\left(\Delta_{\rho}\right): f(z)=\sum_{n=1}^{\infty} a_{n} z^{n}, \quad \sum_{n=1}^{\infty} c_{n}^{-1}\left|a_{n}\right|^{2}<\infty\right\} .
$$

Clearly, this is a Hilbert space of functions $f$, holomorphic in $\Delta_{\rho}, f(0)=0$ and whose norm is

$$
\|f\|_{\phi}=\left\{\sum_{n=1}^{\infty} c_{n}^{-1}\left|a_{n}\right|^{2}\right\}^{1 / 2}
$$

We have

$$
f(\zeta)=\sum_{n=1}^{\infty} a_{n} \zeta^{n}=\sum_{n=1}^{\infty} \frac{a_{n} c_{n} \zeta^{n}}{c_{n}}=(f, \phi(\cdot \bar{\zeta}))_{\phi}
$$

and

$$
|f(\zeta)| \leqq\left\{\phi\left(|\zeta|^{2}\right)\right\}^{1 / 2}\|f\|_{\phi} ; \quad f \in H_{\phi}, \quad \zeta \in \Delta_{\rho},
$$

which means that

$$
k(z, \zeta)=\phi(z \bar{\zeta}) ; \quad z, \zeta \in \Delta_{\rho},
$$

is the reproducing kernel of $H_{\dot{\phi}}$ and that $\left\{\phi_{n}\right\}_{n=1}^{\infty}$ with $\phi_{n}(z)=\sqrt{c_{n}} z^{n}$ is a complete orthonormal system for $H_{\phi}$.

We now consider a new function

$$
\phi(z)=\exp \{\phi(z)\}, \quad z \in \Delta_{\rho} .
$$

Clearly, $\phi \in H\left(\Delta_{\rho}\right)$ and the coefficients of its expansion

$$
\phi(z)=\sum_{n=0}^{\infty} d_{n} z^{n}
$$

satisfy

$$
d_{0}=1, \quad d_{n}=\frac{1}{n} \sum_{k=1}^{n} k c_{k} d_{n-k}, \quad n \geqq 1 .
$$

This shows that $d_{n}>0$ for all $n \geqq 0$ and, in particular

$$
\begin{gathered}
d_{1}=c_{1}, \\
d_{2}=\frac{1}{2 !} c_{1}^{2}+c_{2}, \\
d_{3}=\frac{1}{3 !} c_{1}^{3}+c_{1} c_{2}+c_{3},
\end{gathered}
$$




$$
d_{4}=\frac{1}{4 !} c_{1}^{4}+\frac{1}{2 !} c_{1}^{2} c_{2}+c_{1} c_{3}+\frac{1}{2 !} c_{2}^{2}+c_{4}
$$

and so on. In general,

$$
d_{n}=\sum_{k=1}^{\infty} \frac{1}{k !}\left(\sum_{n_{1}+\cdots+n_{k}=n} c_{n_{1}} \cdots c_{n_{k}}\right), \quad n \geqq 1
$$

where $n_{j} \geqq 1, \jmath=1, \cdots, k$, are integers. This show that $d_{n} \equiv d_{n}\left(c_{1}, \cdots, c_{n}\right), n \geqq 1$, is a polynomial of degree $n$ in $c_{1}, \cdots, c_{n}$ and of rational coefficients. Moreover, for any $\lambda \in \boldsymbol{C}$, we have

$$
d_{n}\left(\lambda c_{1}, \cdots, \lambda^{n} c_{n}\right)=\lambda^{n} d_{n}\left(c_{1}, \cdots, c_{n}\right), \quad n \geqq 1 .
$$

Now, the new function $\phi$ determines another Hilbert space $H_{\psi}$ of functions $g$,

$$
g(z)=\sum_{n=0}^{\infty} b_{n} z^{n}, \quad z \in \Delta_{\rho},
$$

holomorphic in $\Delta_{\rho}$, with $\|g\|_{\psi}<\infty$ where

$$
\|g\|_{\psi}=\left\{\sum_{n=0}^{\infty} d_{n}^{-1}\left|b_{n}\right|^{2}\right\}^{1 / 2} .
$$

As before, this Hilbert space has

$$
K(z, \zeta)=\phi(z \bar{\zeta})=\exp \{\phi(z \bar{\zeta})\} ; \quad z, \zeta \in \Delta_{\rho},
$$

as its reproducing kernel. We also note that, as in the case of $\phi, \Delta_{\rho}$ is the domain of holomorphy of $\psi$.

The main theorem in this direction is:

Theorem 1. Let $f \in H_{\phi}$, then $\exp f \in H_{\phi}$ and

$$
\|\exp f\|_{\psi}^{2} \leqq \exp \|f\|_{\phi}^{2}
$$

with equality if and only if $f=k(\cdot, \zeta)$ for some $\zeta \in \Delta_{\rho}$. In other words, if and only if $f$ is of the form

for some $\zeta \in \Delta_{\rho}$.

$$
f(z)=\phi(z \bar{\zeta}), \quad z \in \Delta_{\rho}
$$

In order to prove this theorem we make the following observation: Let $f \in H_{\phi}$ with

$$
f(z)=\sum_{n=1}^{\infty} a_{n} z^{n}, \quad z \in \Delta_{\rho}
$$

and consider its exponential transform

$$
g(z)=\exp \{f(z)\}=\sum_{n=0}^{\infty} b_{n} z^{n}, \quad z \in \Delta_{\rho} .
$$


Then, as in (2.4),

$$
b_{0}=1, \quad b_{n}=\frac{1}{n} \sum_{k=1}^{n} k a_{k} b_{n-k}, \quad n \geqq 1 .
$$

In view of (2.1)-(2.9), Theorem 1 is, therefore, completely equivalent to the following lemma:

LEMMA 1. Let the notation of (2.1)-(2.9) apply. Then

$$
\sum_{n=0}^{\infty} d_{n}^{-1}\left|b_{n}\right|^{2} \leqq \exp \left\{\sum_{n=1}^{\infty} c_{n}^{-1}\left|a_{n}\right|^{2}\right\}
$$

if the right hand side is finite. Equality holds if and only if $a_{n}=c_{n} \bar{\zeta}^{n}(n \geqq 1)$ and $b_{n}=d_{n} \bar{\zeta}^{n}(n \geqq 0)$ for some $\zeta \in \Delta_{\rho}$.

Proof. For $r \in[0,1)$, define

$$
A(r)=\sum_{n=1}^{\infty} c_{n}^{-1}\left|a_{n}\right|^{2} r^{n}, \quad B(r)=\sum_{n=0}^{\infty} d_{n}^{-1}\left|b_{n}\right|^{2} \gamma^{n} .
$$

Using (2.4), (2.9) and Cauchy-Schwarz inequality

$$
\begin{aligned}
\left|b_{n}\right|^{2} & =\frac{1}{n^{2}}-\left|\sum_{k=1}^{n}\left\{\frac{k}{c_{k} d_{n-k}}\right\}^{1 / 2} a_{k} b_{n-k}\left\{k c_{k} d_{n-k}\right\}^{1 / 2}\right|^{2} \\
& \leqq \frac{1}{n^{2}}\left(\sum_{k=1}^{n} k \frac{\left|a_{k}\right|^{2}}{c_{k}} \frac{\left|b_{n-k}\right|^{2}}{d_{n-k}}\right)\left(\sum_{k=1}^{n} k c_{k} d_{n-k}\right) \\
& =\left(\sum_{k=1}^{n} k \frac{\left|a_{k}\right|^{2}}{c_{k}} \frac{\left|b_{n-k}\right|^{2}}{d_{n-k}}\right) \frac{1}{n} d_{n}, \quad(n \geqq 1),
\end{aligned}
$$

and thus

$$
n d_{n}^{-1}\left|b_{n}\right|^{2} \leqq \sum_{k=1}^{n} k \frac{\left|a_{k}\right|^{2}}{c_{k}} \frac{\left|b_{n-k}\right|^{2}}{d_{n-k}} .
$$

This shows that $B^{\prime}(r) \leqq A^{\prime}(r) B(r)$ or $[\log B(r)]^{\prime} \leqq A^{\prime}(r)$. Consequently, since $A(0)=0$ and $B(0)=1$,

$$
\log B(r)=\int_{0}^{r}[\log B(x)]^{\prime} d x \leqq \int_{0}^{r} A^{\prime}(x) d x=A(r)
$$

and (2.10) follows by letting $r \rightarrow 1^{-}$. In view of $[\log B(r)]^{\prime} \leqq A^{\prime}(r), r \in[0,1)$, equality in (2.10) holds if and only if $B^{\prime}(r)=A^{\prime}(r) B(r)$ for each $r \in[0,1)$ which is equivalent to having equality in (2.11) for every $n \geqq 1$. This in turn is equivalent to an existence of $\lambda_{n} \in \boldsymbol{C}$ so that

$$
a_{k} b_{n-k}=\lambda_{n} c_{k} d_{n-k}, \quad k=1,2, \cdots, n .
$$

Putting $k=n$ in (2.12) results in $a_{n}=\lambda_{n} c_{n}$. On the other hand, summing up (2.12) from $k=1$ through $k=n$, and, using (2.4) and (2.9) yields $b_{n}=\lambda_{n} d_{n}$. Con- 
sequently,

$$
a_{n}=c_{n} d_{n}{ }^{-1} b_{n}, \quad n=1,2, \cdots,
$$

and with (2.9) this also shows

$$
a_{n}\left(d_{n}-c_{n}\right)=\frac{1}{n} c_{n} \sum_{k=1}^{n-1}(n-k) d_{k} c_{k}{ }^{-1} a_{k} b_{n-k}, \quad n \geqq 1 .
$$

Now, the only solution of (2.13) subject to (2.4) and (2.9) is

$$
a_{n}=c_{n} \bar{\zeta}^{n}, \quad \bar{\zeta} \equiv a_{1} c_{1}^{-1} ; \quad n=1,2, \cdots,
$$

and, therefore, $b_{n}=d_{n} \bar{\zeta}^{n}$ for $n \geqq 0$. Indeed, for $n=1$, (2.15) is trivially satisfied while for $n \geqq 1$, by (2.14) and the inductive assumption,

$$
a_{n}\left(d_{n}-c_{n}\right)=c_{n} \bar{\zeta}^{n} \frac{1}{n} \sum_{k=1}^{n-1} k c_{k} d_{n-k}, \quad n>1 .
$$

In view of (2.4) this may be written as

$$
a_{n}\left(d_{n}-c_{n}\right)=c_{n} \bar{\zeta}^{n}\left(d_{n}-c_{n}\right), \quad n>1 .
$$

However, by (2.4) we have $d_{n}>c_{n}$ for every $n>1$ and, therefore, $a_{n}=c_{n} \bar{\zeta}^{n}$, and (2.15) follows. Finally, the right hand side of (2.10) for this solution

$$
\exp \left\{\sum_{n=1}^{\infty} c_{n}|\zeta|^{2 n}\right\}=\exp \left\{\phi\left(|\zeta|^{2}\right)\right\}
$$

is assumed to be finite. Since the disk of convergence of $\phi$ is $\Delta_{\rho}(\rho=1, \infty)$ we must have $\zeta \in \Delta_{\rho}$. This concludes the proof of the lemma.

\section{§3. Applications.}

Many interesting norm-inequalities may be deduced from Theorem 1. However, before so doing we introduce some notation from the theory of hypergeometric and confluent hypergeometric functions.

In this paper, $\alpha, \beta, \gamma, \delta, \mu, p$ and $q$ are real positive numbers. By $(\alpha)_{n}$ we mean $(\alpha)_{0}=1$ and $(\alpha)_{n}=\alpha(\alpha+1) \cdots(\alpha+n-1)$ for $n \geqq 1$. An alternative definition for $(\alpha)_{n}$ is $(\alpha)_{n}=\Gamma(\alpha+n) / \Gamma(\alpha)$. The function

$$
F_{p}(\alpha, \beta ; \gamma: z) \equiv \frac{\Gamma(\gamma)}{\Gamma(\alpha) \Gamma(\beta)} \sum_{n=0}^{\infty} \frac{\Gamma(\alpha+n p) \Gamma(\beta+n p)}{\Gamma(\gamma+n p) \Gamma(1+n p)} z^{n}
$$

is holomorphic in the unit disk $A$, which also forms its disk of convergence. In particular,

$$
F_{1}(\alpha, \beta ; \gamma: z)={ }_{2} F_{1}(\alpha, \beta ; \gamma: z)=\sum_{n=0}^{\infty} \cdot \frac{(\alpha)_{n}(\beta)_{n}}{\left(\gamma_{n}\right)}-\frac{1}{n !} z^{n}
$$

is the familiar hypergeometric function. Moreover, if $p$ is an integer, then 


$$
F_{p}(\alpha, \beta ; \gamma: z)=\sum_{n=0}^{\infty} \frac{(\alpha)_{n p}(\beta)_{n p}}{(\gamma)_{n p}} \frac{1}{(n p) !} z^{n}
$$

and therefore

$$
F_{p}(\alpha, \beta ; \gamma: z)=\frac{1}{p} \sum_{k=0}^{p-1}{ }_{2} F_{1}\left(\alpha, \beta ; \gamma: \omega^{k} z^{1 / p}\right)
$$

with

$$
\omega \equiv e^{2 \pi \imath / p}, \quad p=1,2, \cdots .
$$

We define $F_{\alpha, p}(z) \equiv F_{p}(\alpha, \beta ; \beta: z)$; hence

$$
F_{\alpha, p}(z)=\frac{1}{p} \sum_{k=0}^{p-1}\left(1-\omega^{k} z^{1 / p}\right)^{-\alpha}
$$

where $p$ and $\omega$ are as in (3.3). In particular,

$$
\begin{gathered}
F_{\alpha, 1}(z)=(1-z)^{-\alpha}, \\
F_{\alpha, 2}(z)=\frac{1}{2}\left\{(1-\sqrt{z})^{-\alpha}+(1+\sqrt{z})^{-\alpha}\right\} .
\end{gathered}
$$

We also note that $F_{1, p}(z)=(1-z)^{-1}$, independently of $p$.

The well-known integral representation

$$
{ }_{2} F_{1}(\alpha, \beta ; \gamma: z)=\frac{\Gamma(\gamma)}{\Gamma(\beta) \Gamma(\gamma-\beta)} \int_{0}^{1}(1-t z)^{-\alpha} t^{\beta-1}(1-t)^{\gamma-\beta-1} d t \quad(\gamma>\beta)
$$

when applied to (3.1) and (3.3) induces the generalization

$$
F_{p}(\alpha, \beta ; \gamma: z)=\frac{\Gamma(\gamma)}{\Gamma(\beta) \Gamma(\gamma-\beta)} \int_{0}^{1} F_{\alpha, p}\left(t^{p} z\right) t^{\beta-1}(1-t)^{\gamma-\beta-1} d t
$$

for $\gamma>\beta$ and $p=1,2, \cdots$.

Another related function that we shall consider is

$$
G_{\alpha, p}(z) \equiv z F_{p}(\alpha+p ; 1 ; 1+p: z), \quad z \in \Delta .
$$

This function can be also extended to the case when $\alpha=0$. In fact,

$$
G_{0, p}(z) \equiv z F_{p}(p, 1 ; 1+p: z)=-\log (1-z), \quad z \in \Delta,
$$

independently of $p$. Of course,

$$
G_{\alpha, p}(z)=p B(\alpha, p)\left\{F_{\alpha, p}(z)-1\right\}, \quad z \in \Delta,
$$

where

Evidently,

$$
B(\alpha, p) \equiv \Gamma(\alpha) \Gamma(p) / \Gamma(\alpha+p)
$$

$$
\lim _{\alpha \rightarrow 0} G_{\alpha, p}(z)=G_{0, p}(z)=-\log (1-z)
$$


Moreover, if $p$ is an integer,

$$
G_{\alpha, p}(z)=\frac{p !}{(\alpha)_{p}}\left\{\frac{1}{p} \sum_{k=0}^{p-1}\left(1-\omega^{k} z^{1 / p}\right)^{-\alpha}-1\right\} \quad\left(\omega=e^{2 \pi \imath / p}\right) .
$$

In particular,

$$
G_{\alpha, 1}(z)=\frac{1}{\alpha}\left\{(1-z)^{-\alpha}-1\right\}
$$

and

$$
G_{\alpha, 2}(z)=\frac{1}{\alpha(\alpha+1)}\left\{(1-\sqrt{z})^{-\alpha}+(1+\sqrt{z})^{-\alpha}-2\right\} .
$$

We now turn to the entire functions

$$
\begin{gathered}
E_{p}(\alpha, \beta ; \gamma, \delta: z) \equiv \frac{\Gamma(\gamma) \Gamma(\delta)}{\Gamma(\alpha) \Gamma(\beta)} \sum_{n=0}^{\infty} \frac{\Gamma(\alpha+n p) \Gamma(\beta+n p)}{\Gamma(\gamma+n p) \Gamma(\delta+n p)} \frac{z^{n}}{\Gamma(1+n p)}, \\
E_{p}(\alpha ; \beta: z) \equiv E_{p}(\alpha, r ; \gamma, \beta: z)
\end{gathered}
$$

and

$$
E_{\alpha, p}(z) \equiv E_{p}(1 ; \alpha: z) .
$$

Again, when $p$ is an integer, we have

$$
\begin{gathered}
E_{p}(\alpha, \beta ; \gamma, \delta: z)=\frac{1}{p} \sum_{k=0}^{p-1}{ }_{2} F_{2}\left(\alpha, \beta ; \gamma, \delta: \omega^{k} z^{1 / p}\right), \\
E_{p}(\alpha ; \beta: z)=\frac{1}{p} \sum_{k=0}^{p-1}{ }_{1} F_{1}\left(\alpha ; \beta: \omega^{k} z^{1 / p}\right)
\end{gathered}
$$

and

$$
E_{\alpha, p}(z)=\frac{1}{p} \sum_{k=0}^{p-1}{ }_{1} F_{1}\left(1 ; \alpha: \omega^{k} z^{1 / p}\right) \quad\left(\omega=e^{2 \pi \imath / p}\right),
$$

where ${ }_{2} F_{2}$ and ${ }_{1} F_{1}$ are the familiar confluent hypergeometric functions. Thus

$$
E_{1, p}(z)=\frac{1}{p} \sum_{k=0}^{p-1} e^{\omega^{k_{z} 1 / p}} \quad\left(\omega=e^{2 \pi \imath / p}, \quad p=1,2, \cdots\right) .
$$

In particular

$$
E_{1,1}(z)=e^{z}, \quad E_{1,2}(z)=\cosh \sqrt{z} .
$$

The well-known integral representation

$$
{ }_{1} F_{1}(\alpha ; \beta: z)=\frac{\Gamma(\beta)}{\Gamma(\alpha)} \Gamma(\bar{\beta}-\alpha) \int_{0}^{1} e^{t z} t^{\alpha-1}(1-t)^{\beta-\alpha-1} d t \quad(\beta>\alpha),
$$

when applied to (3.11) and (3.12), induces the generalizations 


$$
E_{p}(\alpha ; \beta: z)=\frac{\Gamma(\beta)}{\Gamma(\alpha) \Gamma(\beta-\alpha)} \int_{0}^{1} E_{1, p}\left(t^{p} z\right) t^{\alpha-1}(1-t)^{\beta-\alpha-1} d t \quad(\beta>\alpha)
$$

and

$$
E_{\alpha, p}(z)=(\alpha-1) \int_{0}^{1} E_{1, p}\left(t^{p} z\right)(1-t)^{\alpha-2} d t \quad(\alpha>1)
$$

for $p=1,2, \cdots$. In particular,

$$
\begin{aligned}
& E_{2}(\alpha ; \beta: z)=\frac{\Gamma(\beta)}{\Gamma(\alpha) \Gamma(\beta-\alpha)} \int_{0}^{1} \cosh (t \sqrt{z}) t^{\alpha-1}(1-t)^{\beta-\alpha-1} d t \quad(\beta>\alpha), \\
& E_{2, p}(z)=\frac{1}{p z^{1 / p}} \sum_{k=0}^{p-1} \omega^{-k}\left[e^{\omega^{k_{2} 1 / p}}-1\right] \quad\left(\omega=e^{2 \pi \nu / p}, \quad p=1,2, \cdots\right),
\end{aligned}
$$

and so

and

$$
E_{2,1}(z)=z^{-1}\left(e^{z}-1\right), \quad E_{2,2}(z)=\sinh \sqrt{z} / \sqrt{z}
$$

$$
E_{\alpha, 2}(z)=(\alpha-1) \int_{0}^{1} \cosh (t \sqrt{z})(1-t)^{\alpha-2} d t \quad(\alpha>1) .
$$

Finally, let $f \in H\left(\Delta_{\rho}\right)$ be given by

$$
f(z)=\sum_{n=0}^{\infty} a_{n} z^{n}, \quad z \in \Delta_{\rho}
$$

and let $r \in[0,1]$. The " $r$-th fractional derivative" of $f$ is

$$
f<r>(z) \equiv \sum_{n=0}^{\infty} n^{r} a_{n} z^{n-1+[1-r]}, \quad z \in \Delta_{\rho},
$$

where $[1-r]$ stands for the integer value of $1-r$ (i. e. 1 if $r=0$ and 0 if $0<r \leqq 1$ ). Clearly, $f^{\langle r\rangle} \in H\left(\Delta_{\rho}\right)$, and $f^{<0>}=f$ and $f^{<1>}=f^{\prime}$. We shall also write $f^{+}$for $f^{<1 / 2\rangle}$, that is

$$
f^{+}(z) \equiv f^{<1 / 2>}(z)=\sum_{n-1}^{\infty} n^{1 / 2} a_{n} z^{n-1}, \quad z \in \Delta_{\rho} .
$$

We now give concrete examples for functions holomorphic in the unit disk and entire functions.

\section{§4. Generalized Hardy Spaces.}

The function $\phi(z) \equiv F_{p}(\alpha, \beta ; \gamma: z)$ generates a reproducing kernel space $\mathscr{H}[p: \alpha, \beta ; \gamma]$ of all functions $f \in H(\Delta)$,

$$
f(z)=\sum_{n=0}^{\infty} a_{n} z^{n}, \quad z \in \Delta,
$$

normed by 


$$
\|f\|_{\mathscr{G}[\bar{p} ; \alpha, \beta ; \gamma]}^{2} \equiv \frac{\Gamma(\alpha) \Gamma(\beta)}{\Gamma(\gamma)} \sum_{n=0}^{\infty} \frac{\Gamma(\gamma+n p) \Gamma(1+n p)}{\Gamma(\alpha+n p) \Gamma(\beta+n p)}\left|a_{n}\right|^{2} .
$$

The reproducing kernel of this space is

$$
K(z, \zeta) \equiv F_{p}(\alpha, \beta ; \gamma: z \bar{\zeta}) ; \quad z, \zeta \in \Delta .
$$

In particular, when $\beta=\gamma$, the reproducing kernel is $K(z, \zeta)=F_{\alpha, p}(z \bar{\zeta})$. The corresponding reproducing space of the latter is denoted by $\mathscr{H}_{\alpha, p}$, i. e. $\mathscr{H}_{\alpha, p}=$ $\mathscr{H}[p: \alpha, \beta ; \beta]$, and the corresponding norm by $\|\cdot\|_{\alpha, p}$. The integral representation (3.6) shows that any $\mathscr{T}[p: \alpha, \beta ; \gamma]$, where $p$ is an integer and $\gamma>\beta$, is a weighted direct sum of spaces $\mathscr{T}_{\alpha, p}$ defined on disks $\Delta_{1 / t} \equiv\{z \in C:|z|<1 / t\}$, $0<t<1$. The spaces $\mathscr{H}[p: \alpha, \beta ; \gamma]$ may be regarded as generalizations of Hardy speces (see also $[3,4]$ and $[9])$; in particular, $\mathscr{H}_{\alpha, p}$ will be called the " $(\alpha, p)$ Hardy space". The norm of $\mathscr{H}_{\alpha, p}$ can be realized as

$$
\|f\|_{\alpha, p}^{2}=|f(0)|^{2}+\frac{1}{\pi} \int_{\Delta}\left|f^{+}(z)\right|^{2}\left(1-|z|^{2 / p}\right)^{\alpha-1} d \sigma(z)
$$

where $f^{+}$is the previously defined half-fractional derivative of $f$ and $d \sigma(z)=d x d y$ is the area Lebesgue measure of $\boldsymbol{C}$. In particular, when $\alpha \geqq 1$, we have

$$
\begin{gathered}
\|f\|_{\alpha, p}^{2}=\frac{\alpha-1}{p \pi} \int_{\Delta}|f(z)|^{2}|z|^{2(1-p) / p}\left(1-|z|^{2 / p}\right)^{\alpha-2} d \sigma(z), \quad \alpha>1, \\
\|f\|_{1, p}^{2}=\frac{1}{2 \pi} \int_{\partial \Delta}|f(z)|^{2}|d z|,
\end{gathered}
$$

where in the last integral, $f$ stands for the nontangential boundary values of the holomorphic function $f$ in $\Delta$. Thus, $\mathscr{T}_{1, p} \equiv \mathscr{T}_{1,1}$ (for any $p$ ) is the HardySzegö space with the Szegö kernel $(1-z \bar{\zeta})^{-1}, \mathscr{T}_{2,1}$ is the Bergman-space with the kernel $(1-z \bar{\zeta})^{-2}$, and $\mathscr{H}_{\alpha, 1}, \alpha>1$, is the Bergman-space with the kernel $(1-z \bar{\zeta})^{-\alpha}$. These spaces are well-known and their norms are realized as genuine integrals. Formulae (4.2)-(4.3) show that same is true for all $\mathscr{H}_{\alpha, p}$ with $\alpha \geqq 1$ and $p>0$. In particular, as the reproducing kernel $F_{\alpha, p}(z \bar{\zeta})$ of $\mathscr{H}_{\alpha, p}$, for integer $p$, admits a closed form expression through (3.3), new and concrete representations are obtained. For example, a use of (3.5) and (4.2) shows the following interesting integral representation: Let $\alpha>1$, then for any $f$, holomorphic in $\Delta$ and such that

$$
\int_{\Delta}|f(z)|^{2}|z|^{-1}(1-|z|)^{\alpha-2} d \sigma(z)<\infty
$$

it holds that

$$
f(\zeta)=\frac{\alpha-1}{4 \pi} \int_{\Delta} f(z)\left\{(1-\sqrt{\bar{z} \zeta})^{-\alpha}+(1+\sqrt{\bar{z} \zeta})^{-\alpha}\right\}|z|^{-1}(1-|z|)^{\alpha-2} d \sigma(z)
$$

for all $\zeta \in \Delta$.

Of course, when Theorem 1 is applied to these spaces it induces sharp norm 
inequalities of exponential type. In order to be more concrete, however, we shall also consider the reproducing kernel space $\mathscr{D}_{q, \alpha, p}$ generated by the function $\phi(z)$ $\equiv q G_{\alpha, p}(z)$. In veiw of (3.7) and (4.1), this is a space of functions $f$ in $H(\boldsymbol{\Delta})$, $f(0)=0$ and with the norm

$$
\|f\|_{\mathscr{D}_{q, \alpha, p}}^{2} \equiv \frac{1}{q p B(\alpha, p)}-\|f\|_{\alpha, p}^{2},
$$

and hence

$$
\|f\|_{\mathscr{D}_{q, \alpha}, p}^{2}=\frac{1}{q p B(\alpha, p)} \frac{1}{\pi} \int_{\Delta}\left|f^{+}(z)\right|^{2}\left(1-|z|^{2 / p}\right)^{\alpha-1} d \sigma(z) .
$$

This space can be also extended to include the case of $\alpha=0$. Indeed, for $\alpha=0$ we have the Dirichlet norm

$$
\|f\|_{\mathscr{D}_{q, 0, p}}^{2}=\frac{1}{q \pi} \int_{\Delta}\left|f^{\prime}(z)\right|^{2} d \sigma(z)
$$

independently of $p$. For this reason, the spaces $\mathscr{D}_{q, \alpha, p}$ will be called generalized " $(q, \alpha, p)$-Diruchlet spaces". When $p=1$, we shall write simply $\mathscr{D}_{q, \alpha}$ instead of $\mathscr{D}_{q, \alpha, 1}$ and we note that $\mathscr{D}_{q, 0} \equiv \mathscr{D}_{q, 0, p}$ for any $p$. The reproducing kernel of $\mathscr{D}_{q, \alpha, p}$ is

$$
k_{q, \alpha, p}(z, \zeta)=q p B(\alpha, p)\left\{F_{\alpha, p}(z \bar{\zeta})-1\right\}
$$

In particular, the reproducing kernel of $\mathscr{D}_{q, \alpha}$ is

$$
k_{q, \alpha}(z, \zeta)=q \alpha^{-1}\left\{(1-z \bar{\zeta})^{-\alpha}-1\right\}, \quad \alpha>0
$$

or

$$
k_{q, 0}(z, \zeta)=-q \log (1-z \bar{\zeta}) .
$$

We also note that the norm of $\mathscr{D}_{q, \alpha, p}$ for $\alpha \geqq 1$ can be also realized, in view of (4.4)-(4.5), as a multiple of the integrals (4.2)-(4.3).

We now consider the reproducing kernel space $\mathcal{E}_{q, \alpha, p}$ generated by $\exp \left\{q G_{\alpha, p}(z)\right\}$. Again, we write $\mathcal{E}_{q, \alpha}$ for $\mathcal{E}_{q, \alpha, 1}$. The reproducing kernel of $\mathcal{E}_{q, \alpha, p}$ is $\exp \left\{k_{q, \alpha, p}(z, \zeta)\right\}$, and we note that $\mathcal{E}_{q, 0}$ coincides with the previously defined Hardy space $\mathscr{H}_{q, 1}$ whose reproducing kernel is $(1-z \bar{\zeta})^{-q}$. According to Theorem 1 , if $f \in \mathscr{D}_{q, \alpha, p}$ then $\exp f$ is in $\mathcal{E}_{q, \alpha, p}$ and

$$
\|\exp f\|_{\mathcal{C}^{2}, \alpha, p}^{2} \leqq \exp \|f\|_{\mathscr{D}_{q}, \alpha, p}^{2}
$$

with equality if and only if $f=q G_{\alpha, p}(\cdot \bar{\zeta})$ for some $\zeta \in \Delta$.

A specially interesting case of this result is when $p=1$. In this case

$$
\exp \left\{q G_{\alpha, 1}(z)\right\}=\sum_{m=0}^{\infty} \frac{1}{m !} p_{m}(q: \alpha) z^{m}
$$

where for any $\alpha \geqq 0, p_{m}(q: \alpha)>0$ for all $q>0$ and $m \geqq 0$. The coefficients $p_{m}(q)$ $\equiv p_{m}(q: \alpha)$ are polynomials of degree $m$ in $q$, satisfying 
and thus

$$
p_{0}(q)=1, \quad p_{m+1}(q)=(m+q) p_{m}(q)+\alpha q p_{m}^{\prime}(q)
$$

and in general

$$
p_{1}(q)=q, \quad p_{2}(q)=q^{2}+(\alpha+1) q,
$$

$$
p_{m}(q: \alpha)=p_{m}(q)=\sum_{n=1}^{m} \frac{(-1)^{n}}{n !}\left[\sum_{k=1}^{n}(-1)^{k}\left(\begin{array}{l}
n \\
k
\end{array}\right)(\alpha k)_{m}\right]\left(\begin{array}{c}
q \\
\alpha
\end{array}\right)^{n}, \quad m \geqq 1 .
$$

Therefore $p_{m}(q)$ is a monic ploynomial with $p_{m}(0)=0$ for $m \geqq 1$. Moreover, $p_{m}(q: \alpha)$ is, for $m \geqq 1$, a polynomial of degree $m-1$ in $\alpha$ and

$$
p_{m}(q: 0) \equiv(q)_{m} \text {. }
$$

It follows that the norm of $\mathcal{E}_{q, \alpha}$ may be given by

$$
\|g\|_{\mathcal{E}_{q, \alpha}}^{2} \equiv \sum_{m=0}^{\infty} \frac{m}{p_{m}(q: \alpha)}\left|b_{m}\right|^{2}
$$

where

$$
g(z)=\sum_{m=0}^{\infty} b_{m} z^{m}, \quad z \in \Delta .
$$

With these more concrete norms, (4.7) gives the following result: If $f \in \mathscr{D}_{q, \alpha}$, then $\exp f \in \mathcal{E}_{q, \alpha}$ and

$$
\|\exp f\|_{\mathcal{E}_{q}, \alpha}^{2} \leqq \exp \|f\|_{\mathscr{D}_{q, \alpha}}^{2}
$$

with equality if and only if $f=k_{q, \alpha}(\cdot, \zeta)$ for some $\zeta \in \Delta$. We note that in view of (4.4)-(4.6), the norm of $\mathscr{D}_{q, \alpha}$ is $(\alpha / q)^{1 / 2}$ times the Hardy-space norm $\|\cdot\|_{\alpha, 1}$ when $\alpha>0$ and $(1 / q)^{1 / 2}$ times the Dirichlet norm when $\alpha=0$. The special case of this result when $\alpha=0$ appears also in Burbea [4] with a somewhat similar proof. The more special case of $\alpha=0$ but $q \geqq 1$ was proved earliear by Saitoh [9], by using different methods.

\section{$\S 5$. Generalized Fischer Spaces.}

The function $\phi(z)=E_{p}(\alpha, \beta ; \gamma, \delta: \mu z)$ generates a reproducing kernel space $\mathscr{I}[p: \alpha, \beta ; \gamma, \delta: \mu]$ of all functions $f \in H(\boldsymbol{C})$, .

$$
f(z)=\sum_{n=0}^{\infty} a_{n} z^{n}, \quad z \in C,
$$

normed by

$$
\|f\|_{\mathscr{S}[p: \alpha, \beta ; \gamma, \delta, \mu]}^{2} \equiv \frac{\Gamma(\alpha) \Gamma(\beta)}{\Gamma(\gamma) \Gamma(\delta)} \sum_{n=0}^{\infty} \frac{\Gamma(\gamma+u p) \Gamma(\delta+n p)}{\Gamma(\alpha+n p) \Gamma(\beta+n p)} \frac{\Gamma(1+n p)}{\mu^{n}}\left|a_{n}\right|^{2} .
$$

The reproducing kernel of this space is

$$
K(z, \zeta) \equiv E_{p}(\alpha, \beta ; \gamma, \delta: \mu z \bar{\zeta}) ; \quad z, \zeta \in C
$$


These spaces may be regarded as generalizations of Fischer spaces (see also [2], [3], [7] and [10]). We shall also consider particular cases of these spaces, namely $\mathscr{F}_{p}(\alpha ; \beta: \mu) \equiv \mathscr{F}[p: \alpha, \gamma ; \gamma, \beta: \mu], \mathscr{F}_{p}(\alpha: \mu) \equiv \mathscr{F}_{p}(1 ; \alpha: \mu)$ and $\mathscr{T}_{p}(\alpha ; \beta: \mu)$ $\equiv \mathscr{F}[p: \alpha, 1 ; \alpha+1, \beta: \mu]$ with the reproducing kernels $E_{p}(\alpha ; \beta: \mu z \bar{\zeta}), E_{\alpha, p}(\mu z \bar{\zeta})$ and $E_{p}(\alpha, 1 ; \alpha+1, \beta: \mu z \bar{\zeta})$, respectively. The integral representation (3.13) shows that any $\mathscr{T}_{p}(\alpha ; \beta: \mu)$, where $p$ is an integer and $\beta>\alpha$, is a weighted direct sum of spaces $\mathscr{F}_{p}(1: s \mu), 0<s<1$, with the weight $p^{-1} s^{\alpha / p-1}\left(1-s^{1 / p}\right)^{\beta-\alpha-1}$. The reproducing kernel of $\mathscr{T}_{p}(1: s \mu)$ is, of course, $E_{1, p}(\mu s z \bar{\zeta})$ which may be evaluated by (3.12).

The norm of $\mathscr{F}_{p}(\alpha: \mu)$ can be realized as

$$
\|f\|_{\mathscr{I}_{p}(\alpha ; \mu)}^{2}=\frac{\mu^{\alpha / p}}{p \pi \Gamma(\alpha)} \int_{C}|f(z)|^{2}|z|^{2(\alpha / p-1)} e^{-\left(\mu|z|^{2}\right)^{1 / p}} d \sigma(z) .
$$

In fact, we shall verify directly that (5.2) induces a reproducing kernel space $\mathscr{F}_{p}(\alpha: \mu)$ of entire functions with the reproducing kernel $E_{\alpha, p}(\mu z \bar{\zeta})$. Let $0<r<\infty$ and define $\Delta_{r}=\{z \in C:|z|<r\}$. Consider

$$
M_{f}(r) \equiv \frac{\mu^{\alpha / p}}{p \pi \Gamma(\alpha)} \int_{\Delta_{r}}|f(z)|^{2}|z|^{2(\alpha / p-1)} e^{-\left(\mu|z|^{2}\right)^{1 / p}} d \sigma(z)
$$

Then

$$
\|f\|_{\mathscr{F}_{p}(\alpha: \mu)}^{2}=\lim _{r \rightarrow \infty} M_{f}(r)
$$

and for $f \in \mathscr{F}_{p}(\alpha: \mu)$ given by (5.1),

$$
M_{f}(r)=\frac{1}{\Gamma(\alpha)} \sum_{n=0}^{\infty} \frac{\Gamma(\mu+n p)}{\mu^{n}}\left|a_{n}\right|^{2} \gamma_{n}(r)
$$

with

$$
\gamma_{n}(r)=\frac{1}{\Gamma(\alpha+n p)} \int_{0}^{(\mu r 2) 1 / p} e^{-\rho} \rho^{\alpha+n p-1} d \rho .
$$

It follows that (i) $0<\gamma_{n}(r)<1$, (ii) $\gamma_{n}(r)$ is a continuously increasing function of $r$ and (iii) $\lim _{r \rightarrow \infty} \gamma_{n}(r)=1$. From these properties it follows (an application of B.

Levi's theorem) that

$$
\|f\|_{\mathscr{F}_{p}(\alpha: \mu)}^{2}=\frac{1}{\Gamma(\alpha)} \sum_{n=0}^{\infty} \frac{\Gamma(\alpha+n p)}{\mu^{n}}\left|a_{n}\right|^{2} .
$$

Moreover, by Cauchy-Schwarz inequality

$$
|f(z)|^{2}=\left|\sum_{n=0}^{\infty} a_{n} z^{n}\right|^{2} \leqq\left(\sum_{n=0}^{\infty} \frac{\Gamma(\alpha+n p)}{\mu^{n}}\left|a_{n}\right|^{2}\right)\left(\sum_{n=0}^{\infty} \frac{\mu^{n}|z|^{2 n}}{\Gamma(\alpha+n p)}\right)
$$

and thus by (3.8)-(3.10) and (5.3)

$$
|f(z)| \leqq\left\{E_{\alpha, p}\left(\mu|z|^{2}\right)\right\}^{1 / 2}\|f\|_{\mathscr{F} p^{(\alpha \cdot \mu)}} .
$$

This shows that $\mathscr{I}_{p}(\alpha: \mu)$ is a Hilbert space with a reproducing kernel $K(\cdot, \cdot)$. 
Now the sequence

$$
\phi_{n}(z)=\left\{\frac{\Gamma(\alpha)}{\Gamma(\alpha+n p)} \mu^{n}\right\}^{1 / 2} z^{n}, \quad n=0,1, \cdots,
$$

is clearly orthonormal in $\mathscr{F}_{p}(\alpha: \mu)$ and by (5.3) it is also complete. Therefore

$$
K(z, \zeta)=\sum_{n=0}^{\infty} \phi_{n}(z) \overline{\phi_{n}(\zeta)}=E_{\alpha, p}(\mu z \bar{\zeta})
$$

and our assertions follow.

The norm-integral (5.2) induces many interesting concrete integral representations for entire functions, some of which are by now classical. For example, the space $\mathscr{F}_{1}(1: \mu)$ is the ordinary Fischer space [7] with the reproducing kernel $E_{1,1}(\mu z \bar{\zeta}) \equiv e^{\mu z \bar{\zeta}}$ and hence the spaces $\mathscr{F}_{p}(\alpha: \mu)$ constitute concrete extensions. In particular, new representation formulae are obtained with $\mathscr{I}_{1}(\alpha: \mu)$ and $E_{\alpha, 1}(\mu z \bar{\zeta})$ $\equiv_{1} F_{1}(1 ; \alpha: \mu z \bar{\zeta})$, with $\mathscr{I}_{1}(2: \mu)$ and $E_{2,1}(\mu z \bar{\zeta})=[\mu z \bar{\zeta}]^{-1}\left(e^{\mu z \bar{\zeta}}-1\right)$, with $\mathscr{F}_{2}(\alpha: \mu)$ and $E_{\alpha, 2}(\mu z \bar{\zeta}) \equiv 2^{-1}\left\{F_{1}(1 ; \alpha: \sqrt{\mu z \bar{\zeta}})+{ }_{1} F_{1}(1 ; \alpha:-\sqrt{\mu z \bar{\zeta}})\right\}$, with $\mathscr{T}_{2}(1 ; \mu)$ and $E_{1,2}(\mu z \bar{\zeta})$ $=\cosh \sqrt{\mu z \bar{\zeta}}$, with $\mathscr{I}_{2}(2: \mu)$ and $E_{2,2}(\mu z \bar{\zeta})=\sinh \sqrt{\mu z \bar{\zeta}} / \sqrt{\mu z \bar{\zeta}}$, and so on, in view of (3.13)-(3.14).

The norm of the related Fischer space $\tilde{F}_{p}(\alpha ; \beta: \mu)$ admits also an integral realization. In fact, the norm is related to the norm of $\mathscr{F}_{p}(\alpha: \mu)$ in (5.2) via

$$
\|f\|_{\widetilde{F}_{p}}^{2}(\alpha ; \beta, \mu)=(\beta / \alpha)\|f\|_{\mathscr{F}_{p}(\beta+1, \mu)}^{2}+(1-\beta / \alpha)\|f\|_{\mathscr{F}_{p}(\beta ; \mu)}^{2}
$$

and hence we may define $\tilde{\mathscr{F}}_{p}(\infty ; \beta: \mu)$ as $\mathscr{T}_{p}(\beta: \mu)$, and $\tilde{\mathscr{T}}_{p}(\alpha ; \alpha: \mu) \equiv \mathscr{F}_{p}(\alpha+1: \mu)$. Notice that the right hand side of (5.3) is non-negative even if $\beta>\alpha$. This can also be shown directly by observing that

$$
\|f\|_{\mathscr{I}_{p}(\beta+1 ; \mu)}^{2}=\|f\|_{\mathscr{F}_{p}(\beta, \mu)}^{2}+\frac{p}{\Gamma(\beta+1)} \sum_{n=1}^{\infty} \frac{\Gamma(\beta+n p)}{\mu^{n}} n\left|a_{n}\right|^{2}
$$

and thus

$$
\|f\|_{\mathscr{F}^{\prime}}^{2} p^{(\beta+1: \mu)} \geqq\|f\|_{\mathscr{F}^{2}}^{2} p^{(\beta ; \mu)}
$$

with equality if and only if $f$ is a constant.

The reproducing kernel $E_{p}(\alpha, 1 ; \alpha+1, \beta ; \mu z \bar{\zeta})$ of $\tilde{\mathscr{T}}_{p}(\alpha ; \beta: \mu)$ is also closely connected with that of $\mathscr{F}_{p}(\alpha: \mu)$. In fact, writing $\tilde{\mathscr{T}}_{p}(\alpha: \mu)$ for $\tilde{\mathscr{T}}_{p}(\alpha ; 1: \mu)$ we see that the reproducing kernel of $\tilde{\mathscr{T}}_{p}(\alpha: \mu)$ becomes $E_{p}(\alpha ; \alpha+1: \mu z \bar{\zeta})$. In particular, when $p$ is an integer it follows from (3.11) that

$$
E_{p}(\alpha ; \alpha+1: \mu z \bar{\zeta})=\frac{1}{p} \sum_{k=1}^{p-1}{ }_{1} F_{1}\left(\alpha ; \alpha+1: \omega^{k}(\mu z \bar{\zeta})^{1 / p}\right) \quad\left(\omega=e^{2 \pi \imath / p}\right) .
$$

On the other hand, applying Kummer's relation

$$
{ }_{1} F_{1}(a ; b: z)=e^{z}{ }_{1} F_{1}(b-a ; b:-z), \quad b>a>0,
$$

yields 


$$
E_{p}(\alpha ; \alpha+1: \mu z \bar{\zeta})=\frac{1}{p} \sum_{k=0}^{p-1} e^{\omega^{k}(\mu z \bar{\zeta}) 1 / p} E_{\alpha+1,1}\left(-\omega^{k}(\mu z \bar{\zeta})^{1 / p}\right)
$$

In particular

$$
E_{1}(\alpha ; \alpha+1: \mu z \bar{\zeta})=e^{\mu z \bar{\zeta}} E_{\alpha+1,1}(-\mu z \bar{\zeta})
$$

These relationships, in view of (5.2) and (5.3), also give new concrete integral representation formulae.

We now consider the space $\mathcal{F}^{0}[p: \alpha, \beta ; \gamma, \delta: \mu]$ consisting of all $f \in$ $\mathscr{F}[p: \alpha, \beta ; \gamma, \delta: \mu]$ with $f(0)=0$. This is a closed subspace of $\mathscr{F}[p: \alpha, \beta ; \gamma, \delta: \mu]$ and, therefore, it is a Hilbert space with the reproducing kernel $k(z, \zeta) \equiv$ $E_{p}(\alpha, \beta ; \gamma, \delta: \mu z \bar{\zeta})-1$. Let $\mathcal{E}[p: \alpha, \beta ; \gamma, \delta: \mu]$ be the Hilbert space generated by

$$
K(z, \zeta)=\exp \{k(z, \zeta)\}=e^{-1} \exp \left\{E_{p}(\alpha, \beta ; \gamma, \delta: \mu z \bar{\zeta})\right\} .
$$

Theorem 1 then shows that if $f \in \mathscr{F}^{0}[p: \alpha, \beta ; \gamma, \delta: \mu]$, then $\exp f$ is in $\mathcal{E}[p: \alpha, \beta ; \gamma, \delta: \mu]$ and

$$
\|\exp f\|_{\subset[p, \alpha, \beta ; \gamma, \delta, \mu]}^{2} \leqq \exp \|f\|_{\mathcal{F}[p, \alpha, \beta ; \gamma, \delta, \mu]}^{2}
$$

with equality if and only if $f$ is of the form $f(z)=E_{p}(\alpha, \beta ; \gamma, \delta: \mu z \bar{\zeta})-1$ for some $\zeta \in C$.

A particularly interesting case occurs for $\mathscr{F}_{\mu} \equiv \mathscr{F}^{0}[1 ; 1,1 ; 1,1: \mu]$ and $\mathcal{E}_{\mu}$ $\equiv \mathcal{E}[1: 1,1 ; 1,1: \mu]$. In this case the reproducing kernels of $\mathscr{F}_{\mu}$ and $\mathcal{E}_{\mu}$ are $k(z, \zeta)$ $=e^{\mu z \bar{\zeta}}-1$ and $K(z, \zeta)=e^{-1} \exp \left\{e^{\mu z \bar{\zeta}}\right\}$, respectively. The norm of $\mathscr{F}_{\mu}$, in view of (5.2), is

$$
\|f\|_{\mu}^{2} \equiv \frac{\mu}{\pi} \int_{C}|f(z)|^{2} e^{-\mu|z|^{2}} d \sigma(z) .
$$

The norm of $\mathcal{E}_{\mu}$ on the other hand is determined from the expansion

$$
e^{-1} \exp \left\{e^{2}\right\}=\sum_{n=0}^{\infty} \frac{s_{n}}{n !} z^{n}
$$

where

$$
s_{n}=e^{-1} \sum_{m=0}^{\infty} \frac{m^{n}}{m !}, \quad n=0,1, \cdots,
$$

are the Stirling numbers of the second kind; $s_{0}=1, s_{1}=1, s_{2}=2, s_{3}=5, s_{4}=15$, $s_{5}=52, s_{6}=203$ and in general

$$
s_{n}=\sum_{m=1}^{n} \frac{(-1)^{m}}{m !}\left(\sum_{m=1}^{m}\left(\begin{array}{c}
m \\
k
\end{array}\right)(-1)^{k} k^{n}\right) \quad(n \geqq 1) .
$$

We also note that $s_{n}$ is the number of different partitions of the set $\{1,2, \cdots, n\}$ and it satisfies the recursion relation

$$
s_{n+1}=\sum_{k=0}^{n}\left(\begin{array}{l}
n \\
k
\end{array}\right) s_{k} .
$$


It follows that

$$
k(z, \zeta)=e^{-1} \exp \left\{e^{\mu z \bar{\zeta}}\right\}=\sum_{n=0}^{\infty} \frac{s_{n}}{n !}(\mu z \bar{\zeta})^{n} ; \quad z, \zeta \in \boldsymbol{C},
$$

and

$$
\|g\|_{\mathcal{C}^{\prime} \mu}^{2}=\sum_{n=0}^{\infty} \frac{n !}{s_{n}} \frac{\left|b_{n}\right|^{2}}{\mu^{n}}
$$

where

$$
g(z)=\sum_{n=0}^{\infty} b_{n} z^{n}, \quad z \in C .
$$

We have therefore shown the following result: Let $f \in \mathscr{F}_{\mu}$, then $\exp f \in \mathcal{E}_{\mu}$ and

$$
\|\exp f\|_{\mathcal{E}_{\mu}}^{2} \leqq \exp \|f\|_{\mu}^{2}
$$

with equality if and only if $f$ is of the form $f(z)=e^{-1} \exp \left\{e^{\mu z \bar{\zeta}}\right\}$ for some $\zeta \in C$.

Acknowledgement: The author is grateful to Professor S. Saitoh for valuable comments on an earlier draft of this paper.

\section{BIBLIOGRAPHY}

[1] Aronszajn, N., Theory of reproducing kernels, Trans. Amer. Math. Soc. 68 (1950), 337-404.

[2] BargmanN, V., On a Hilbert space of analytic functions and an associated integral transform I, Comm. Pure Appl. Math. 14 (1961), 187-214.

[3] Burbea, J., Total positivity of certain reproducing kernels, Pacific J. Math. 67 (1976), 101-130.

[4] Burbea, J., A Dirichlet norm inequality and some inequalitıes for reproducıng kernel spaces, Proc. Amer. Math. Soc. 83 (1981), 279-285.

[5] BurbeA, J., Inequalities for holomorphic functions of several complex variables, Trans. Amer. Math. Soc., to appear.

[6] Milin, I. M., Estimation of coefficients of univalent functions, Dokl. Akad. Nauk. SSSR 160 (1965), 769-771=Soviet Math. Doklady 9 (1968), 762-765.

[7] Newman, D. J. ANd Shapiro, H.S., Certaın Hilbert spaces of entire functions, Bull. Amer. Math. Soc. 72 (1966), 971-977.

[8] Pommerenke, Chr., Univalent Functions, Vandenhoeck and Ruprecht, Göttingen, 1975.

[9] SAIToH, S., Some inequalities for analytic functions with a finite Dirichlet integral on the unit disk, Math. Ann. 246 (1979), 69-77.

[10] SaItoH, S., Some inequalities for entire functions, Proc. Amer. Math. Soc. 80 (1980), 254-258.

Department of Mathematics

University of PitTsBurgh

Pittsburgh, Pennsylvania 15260

U. S. A. 